with further knowledge have to be modified, but $I$ think it cannot be very far from the truth.

In the tree I give, attention may be directed to the placing of Ramapithecus nearer to the base of the human stem than the other Siwalik types, and to the placing of Proconsul higher up than is usually done. Hopwood regards Proconsul as a Lower Miocene form "related to Dryopithecus and ancestral to the chimpanzee". I have only seen the cast, but incline to place it nearer to the gorilla, and appreciably higher. Possibly the supposed Miocene age is wrong.

The reasons for the various conclusions to which I have come will be given at length in the long paper I have in hand on the South African Pleistocene Anthropoids.

\title{
A NEW PROCESS FOR LIQUEFYING AIR
}

\section{By J. H. AwBERY}

$\mathrm{I}^{\mathrm{N}}$

$N$ nearly all refrigeration processes, whether for the moderate cooling associated with focd preservation or for the liquefaction of the 'permanent' gases, the operations are basically similar. A gas or vapour is compressed, the heat due to this compression is removed, and the fluid then allowed to expand, whereby it cools below the temperature of its surroundings. In the Linde process for making liquid air, the compression is to about 200 atmospheres, and the expansion takes place through a nozzle, the resulting cooling being that due to the Joule-Thomson effect; the process is made cumulative by using the cold air to lower the temperature of the air which is still approaching the nozzle. Now the Joule-Thomson effect is by no means large in gases so nearly perfect as air, and it has long been realized that much greater cooling could be obtained in the expansion if this could be carried out in such a way that the gas did work, so that more energy would be removed from it. Mechanical difficulties, such as that of lubrication and of avoiding excessive heating due to friction, have caused designers to favour the theoretically less efficient expansion valve, though Claude had successfully applied the principle as early as 1906 .

In an article in Voks Bulletin (NovemberDecember, 1940), Prof. P. Kapitza gives a brief description of an installation which has now been set up at the Institute of Physical Problems of the Academy of Sciences of the U.S.S.R., in which the expanding air does work by driving a turbine. The increased temperature-fall, as compared with free expansion through a nozzle, is so great that the initial compression need only be of the order of 5 atmospheres, instead of the 200 atmospheres necessary in Linde's process.

The main difficulty to be overcome was the design of the turbine itself, which is quite inefficient if the axial-flow impulse turbine used with steam is taken as a model. Owing to the low temperature, the air flowing through the turbine has a density five times that of steam at $250^{\circ} \mathrm{C}$., so that there is a considerable centrifugal force as the fluid whirls around, and this must be taken into account, just as it is in a water turbine.

The present apparatus, which will be followed by larger ones, has a piston compressor working at 400 r.p.m., taking $50-80 \mathrm{kw}$. of electrical energy and delivering nearly $600 \mathrm{kgm}$. of air per hour at about 7 atmospheres. The compressed air then passes a. water cooler and through the regenerator (cooled by previously treated air) to the turbine. The latter works at 40,000 r.p.m. and yields $4 \mathrm{kwh}$. of mechanical energy. The pressure drop is 4 to 1 , and the cooling is such that air entering at $-158^{\circ} \mathrm{C}$. emerges at $-187^{\circ} \mathrm{C}$. (the boiling point of oxygen), implying the extraction of 3,700 calories per hour. The efficiency is thus $0 \cdot 79-0 \cdot 83$. The regenerators also offer some novel features. There are two of them, used intermittently for about 26 sec. each, the changeover being effected automatically. They are filled with flat ribbon, $0.1 \mathrm{~mm}$. thick and $50 \mathrm{~mm}$. wide, with nodules.

From the turbine, the main air-stream is passed through the inner tubes of a condenser, only a small by-passed stream flowing through the outer tubes and being liquefied therein. The overall efficiency of the apparatus is such that it gives 29-30 kgm. of liquid air per hour, at an energy cost of $1.7 \mathrm{kwh}$. per $\mathrm{kgm}$. It is calculated that this can be reduced to $1.2 \mathrm{kwh}$. per $\mathrm{kgm}$. by making use of the mechanical energy from the turbine and by more suitable valves and other equipment, so as to utilize the full seven atmospheres compression, instead of only four-sevenths of it. If so, the apparatus will have about the same efficiency as present high-pressure installations.

It is even hoped that by working on a much larger scale, where heat losses are relatively smaller, the efficiency may rise to $1 \cdot l$ or even $1.0 \mathrm{kwh}$. per $\mathrm{kgm}$. of liquid air. In any event, as a laboratory installation, the outfit has many advantages, noteworthy among them being the short starting period (20 minutes or less) and the small dimensions, due to absence of decarbonizers, scrubbers, desiccators and other auxiliary gear. 